\title{
DPO multiplex PCR as an alternative to culture and susceptibility testing to detect Helicobacter pylori and its resistance to clarithromycin
}

\author{
Philippe Lehours ${ }^{1,2,3}$, Elodie Siffré ${ }^{1}$ and Francis Mégraud 1,2,3*
}

\begin{abstract}
Background: Macrolide resistance in Helicobacter pylori is the major risk factor for treatment failure when using a proton pump inhibitor-clarithromycin containing therapy. Macrolide resistance is due to a few mutations on the 235 ribomosal subunit encoded by the $23 \mathrm{~S}$ rRNA gene. The present study aimed at investigating the performance of the dual priming oligonucleotide (DPO)-PCR kit named Seeplex ${ }^{\circledR}$ ClaR-H. pylori ACE detection designed to detect H. pylori and two types of point mutations causing clarithromycin resistance in $\mathrm{H}$. pylori.

Methods: The performance of Seeplex ${ }^{\circledR}$ ClaR-H. pylori ACE detection was evaluated on 127 gastric biopsies in comparison to conventional bacterial culture followed by the determination of susceptibility to clarithromycin by E-test, as well as by an in-house real-time PCR using a fluorescence resonance energy transfer (FRET) technology.

Results: Considering culture as the reference test, the sensitivity of DPO-PCR and real-time FRET-PCR was $97.7 \%$ and $100 \%$ while specificity was $83.1 \%$ and $80.7 \%$, respectively. However, both PCR were concordant in detecting 14 H. pylori positive cases which were negative by culture. Globally, E-test and DPO-PCR were concordant with regard to clarithromycin susceptibility in $95.3 \%$ of the cases (41/43), while real-time FRET-PCR and DPO-PCR were concordant in 95\% (57/60).

Conclusion: The DPO-PCR is an interesting tool to detect $H$. pylori on gastric biopsies and to study its susceptibility to clarithromycin in laboratories that cannot perform real-time PCR assays.
\end{abstract}

\section{Background}

Macrolide resistance in Helicobacter pylori is the major risk factor for treatment failure when using a proton pump inhibitor (PPI)-clarithromycin containing therapy [1]. Macrolide resistance is due to a few mutations on the $23 \mathrm{~S}$ ribomosal subunit encoded by the $23 \mathrm{~S}$ rRNA gene $[2,3]$. These mutations (A2142C, A2142G, A2143G), are easy to detect by numerous molecular methods directly on gastric biopsy specimens and even on stool samples [4-7].

A new PCR format named DPO-PCR for "Dual Priming Oligonucleotide" was recently developed [8]. DPOPCR is a multiplex PCR assay that increases specificity and sensitivity of detection compared to conventional PCR, by blocking non-specific binding sites therefore

\footnotetext{
* Correspondence: francis.megraud@chu-bordeaux.fr

'Université de Bordeaux, Centre National de Référence des Campylobacters et des Hélicobacters, 146 rue Léo Saignat, 33000 Bordeaux, France Full list of author information is available at the end of the article
}

eliminating imperfect primer annealing. This new technology can be used for many applications in the field of in vitro diagnostics: simultaneous detection of multiple pathogens and of polymorphisms (SNPs), as well as simultaneous genotyping of multiple pathogen subtypes. DPO-PCR is based on a multiplex PCR using a DPO patented technology [8]. The structure of the DPO primers is fundamentally different from that of conventional primers. Indeed, the primer is divided into two parts by a 5 polydeoxyinosine linker which allows a more specific hybridization at temperatures between 55 and $65^{\circ} \mathrm{C}$. This linker forms a "bubble-like structure" which itself is not involved in priming, rather it delineates the boundary between two parts. It therefore generates two recognition reactions of the primer on the target sequence. According to the manufacturer (see http://www.seegene.com/ en/research/core_020.php), the 5' end (approximately 20 bases) binds preferentially to the matrix and initiates stable annealing acting as a "stabilizer". The 3' end is

\section{C) Biomed Central}


shorter (approximately 10 bases) and binds afterwards to the target site but only if the first step has taken place without a mismatch. The 3' end determines a target-specific extension and acts as a "determiner". Therefore, although the longer 5'-segment binds to a non-target site, the shorter segment resists non-specific extension. The short 3'-portion alone fails to make a priming at an annealing temperature. The latter also binds preferentially to the target and avoids non-specific binding. This PCR can be performed in any conventional thermocycler.

The performance of this PCR format for the detection of $H$. pylori $23 \mathrm{~S}$ rDNA mutations, involved in macrolide resistance was previously evaluated in a study published in 2007 by Woo et al., [9] with a 94.1\% concordance between the DPO-based multiplex PCR and culture followed by a phenotypic susceptibility test.

In an article by Cho AR and Lee MK in Korean language, they also compared this method to culture and histology, and concluded that it could be used for the diagnosis of $H$. pylori infection and the determination of clarithromycin resistance [10]. However, they used a disk diffusion method which is not a generally accepted technique for testing $H$. pylori antimicrobial susceptibility. The present study is a retrospective study performed by the National Reference Centre for Helicobacters in France which aimed at investigating the performance of the Seeplex ${ }^{\circledR}$ ClaR-H. pylori ACE detection kit (Seegene, Seoul, Korea) in comparison to standard phenotypic tests as well as the real-time fluorescence resonance energy transfer (FRET)-PCR developed and routinely used in our laboratory [7].

\section{Methods \\ Materials}

The Seeplex ${ }^{\circledR}$ ClaR-H. pylori ACE detection kit was evaluated retrospectively on DNAs extracted from 127 gastric biopsies received at the French National Reference Centre for Helicobacters (Bordeaux, France) during the year 2009. There was no preselection according to the gastric site. Consecutive biopsies were included until about half of the number of biopsies positive for $H$. pylori was attained.

\section{Methods}

The performance of the kit was compared to conventional bacterial culture followed by the determination of susceptibility to clarithromycin by E-test, and an in-house realtime PCR detection using the FRET technology [7].

\section{Culture}

H. pylori strains were obtained from the corresponding gastric biopsies, after culture on Wilkins-Chalgren agar plates (Oxoid, Dardilly, France) supplemented with human blood $(10 \% \mathrm{v} / \mathrm{v})$ and antibiotics $(10 \mu \mathrm{g} / \mathrm{ml}$ of vancomycin, $10 \mu \mathrm{g} / \mathrm{ml}$ of cefsulodin, $5 \mu \mathrm{g} / \mathrm{ml}$ of trimethoprim, and
$10 \mu \mathrm{g} / \mathrm{ml}$ of amphotericin B) under microaerobic conditions, as already described $[11,12]$. Forty-four culture positive cases were finally included.

\section{Phenotypic susceptibility testing}

Susceptibility to clarithromycin was assessed using the E-test method (bioMérieux, Marcy l'Etoile, France) performed as previously described [6] and using the EUCAST breakpoints: $\mathrm{S} \leq 0.25 \mu \mathrm{g} / \mathrm{ml} ; \mathrm{R}>0.5 \mu \mathrm{g} / \mathrm{ml}$ (http://www.eucast.org/clinical_breakpoints/).

\section{DNA extraction from gastric biopsies}

Genomic DNA from gastric biopsies was extracted by using the MagnaPure LC DNA Isolation Kit I and the MagnaPure LC Isolation Station (Roche Applied Science, Penzberg, Germany). DNAs were stored at $-20^{\circ} \mathrm{C}$ until required for analysis.

\section{Real-time FRET-PCR}

The real-time FRET-PCR is designed to detect clarithromycin susceptible $H$. pylori (wild type) and the mutations responsible for clarithromycin resistance: A4142G and A2143G, without distinguishing between them, as well as A2142C. This test was performed as previously described [7].

\section{DPO-PCR}

DPO-PCR was performed using the Seeplex ${ }^{\circledR}$ ClaR- $H$. pylori ACE detection kit according to the manufacturer's recommendations (Seegene distributed by Eurobio Laboratoires, Courtaboeuf, France) and analyzed using a semi-automated system called Screen tape ${ }^{\circledR}$ allowing an ultra rapid migration and analysis of the PCR products in small polyacrylamide gels. 8-methoxysporalen was added during the mix preparation to intercalate between double-stranded nucleic acids generated during amplification, thereby limiting carry-over contamination after UV irradiation and before PCR product analysis. The Seeplex ${ }^{\circledR}$ ClaR- $H$. pylori ACE detection kit includes 3 primer pairs with a DPO structure which allows amplification of the $H$. pylori $23 \mathrm{~S}$ rDNA (621 bp amplicon) and detection of A2142G and A2143G mutations (194 bp and 475 bp, respectively). The kit also includes a primer pair for internal control.

DPO-PCR is a multiplex PCR that can be performed in any standard thermocycler.

\section{Histology}

Briefly, histological preparations were stained with hematoxylin and eosin and Giemsa stains and the presence of $H$. pylori was evaluated according to the Sydney system. Histological results were used only in case of discrepant results obtained between DPO-PCR and FRET-PCR. 


\section{Evaluation of Sensitivity and Specificity}

The proportion of positives by DPO-PCR among the true positives defined the sensitivity and the proportion of negatives by DPO-PCR among the true negatives defined the specificity.

\section{Results}

Concerning the 127 biopsies included in the study, culture was positive for H. pylori in 44 cases (34.6\%), the real-time FRET-PCR in 60 cases (47.2\%), and the DPOPCR in 57 cases (44.9\%). H. pylori status obtained from histological diagnosis was available for only 89 patients.

For the 44 biopsies positive by culture, the real-time FRET-PCR and DPO-PCR were also positive, except in one case where DPO-PCR was solely negative. For 67 biopsies, culture, FRET-PCR and DPO-PCR were all negative. Out of 16 biopsies negative by culture, FRETPCR, DPO-PCR and histology were all positive for 14 . For the remaining 2 biopsies, real-time FRET-PCR was solely positive (Tables 1 and 2 ) and histology confirmed the presence of $H$. pylori.

Using culture as the reference test, the sensitivity of DPO-PCR and real-time FRET PCR was $97.7 \%$ and $100 \%$, respectively, and the specificity was $83.1 \%$ and $80.7 \%$, respectively.

The concordance between real-time FRET-PCR and DPO-PCR in our study was 95\% (57/60) (Table 2). Considering the $44 \mathrm{H}$. pylori strains isolated by culture, clarithromycin susceptibility results were available for 43 (one strain being lost after subculture). Table 2 summarizes the results obtained by the three methods used for determining macrolide susceptibility. For the 17 clarithromycin susceptible isolates, 15 corresponding biopsies contained a wild type isolate in both PCR formats. One biopsy was categorized as wild type by FRET-PCR and as an A2143G mutant by DPO-PCR. The remaining biopsy was considered to be a mixture of wild type and A2142G/A2143G mutant by FRET-PCR whereas DPOPCR detected an A2143G mutation.

For the 26 macrolide resistant isolates, a $23 \mathrm{~S}$ rDNA mutation was detected in 25 biopsies by both PCR formats. One biopsy was considered as a wild type by realtime FRET-PCR but was negative by DPO-PCR (histology was also negative).
Globally, E-test and DPO-PCR were concordant in $95.3 \%$ of these cases $(41 / 43)$.

\section{Discussion}

We found a good correlation for the detection of H. pylori and the detection of clarithromycin susceptibility between the DPO-PCR and the real-time FRET-PCR routinely used in our Reference Centre. Overall, the performance is very good for a non-real-time PCR format. Compared to other PCR formats developed to detect mutations involved in macrolide resistance for H. pylori, DPO-PCR requires no investment in additional technical or expensive detection devices. One disadvantage is that users must run the detection of PCR fragments themselves on a $2 \%$ agarose gel before analyzing the PCR bands obtained, compared to real-time PCR formats available to date where PCR amplification is monitored automatically. DPO-PCR is therefore more time-consuming. However, in our study the semi-automated system called ScreenTape ${ }^{\circledR}$ was used. ScreenTape ${ }^{\circledR}$ simplifies the analysis of the results of this multiplex PCR assay.

The cost of the test is highly dependent on the activity and equipment of the laboratory in which the test is performed; however, it is significantly higher than the cost of the in-house method tested in parallel in the present study.

DPO-PCR detected more H. pylori positive biopsies than culture alone, with an excellent correlation with the FRET-PCR. Woo et al., identified $49 \mathrm{H}$. pylori positive samples among 165 culture-negative specimens using DPO-PCR [9]. This result leads us to believe that there is no specificity problem regarding DPO-PCR, rather a problem of sensitivity regarding culture.

The excellent correlation between DPO-PCR and E-test susceptibility is in line with the previous study published by Woo et al., where they found a $94.1 \%$ concordance between both methods [9]. In the work of Woo et al., two strains categorized as susceptible by E-test appeared resistant by DPO-PCR. Cho et al., also described that the results of PCR and E-test on 3 of the 8 mutation-positive biopsies were discrepant [10]. In the present study, DPOPCR detected resistances missed by E-test also in two cases (Table 2). This could be explained by the detection

Table 1 Global results obtained for different diagnosis tests for the detection of Helicobacter pylori in human gastric biopsies

\begin{tabular}{llll}
\hline Culture $(\mathbf{n}=\mathbf{4 4})$ & FRET-PCR $(\mathbf{n}=60)$ & DPO-PCR $(\mathbf{n}=\mathbf{5 7})$ & Total $(\mathbf{n}=\mathbf{1 2 7})$ \\
\hline$(+)$ & $(+)$ & $(+)$ & 43 \\
$(+)$ & $(+)$ & $(-)$ & 1 \\
$(-)$ & $(-)$ & $(-)$ & 67 \\
$(-)$ & $(+)$ & $(+)$ & 14 \\
$(-)$ & $(+)$ & $(-)$ & 2 \\
\hline
\end{tabular}

The numbers indicated in parentheses represent the total number of positive samples for each test. 
Table 2 Comparison of Helicobacter pylori susceptibility to clarithromycin by E-test, real-time FRET-PCR and DPO-PCR on human gastric biopsies

\begin{tabular}{llll}
\hline E-test & FRET-PCR & DPO-PCR & Total $(\mathbf{n}=\mathbf{6 0})$ \\
\hline$R$ & $R^{*}$ & $R^{\text {}}$ & 25 \\
WT & WT & WT & 15 \\
WT & $R^{\mu}$ & $R$ & 1 \\
WT & WT & $R$ & 1 \\
$R$ & $W T$ & NEG & 1 \\
ND & $R$ & $R$ & 4 \\
ND & WT & WT & 11 \\
ND & WT & NEG & $2^{\S}$ \\
\hline
\end{tabular}

R: macrolide resistant strain; WT: wild type (macrolide susceptible strain); NEG: negative result; ND: not determined.

* 3 biopsies with a mixture of a wild type and A2142G/A2143G mutants.

$\mu 1$ biopsy with a mixture of a wild type and A2142G/A2143G mutants.

\$ 1 double population A2142G + A2143G.

$\S$ positive histology.

limit of the Seeplex ${ }^{\circledR}$ ClaR-H. pylori ACE detection kit which is 100 copies/reaction (100 copies/3 $\mu$ l DNA). According to Woo HY et al., DPO-PCR can detect mutants present among wild-type strains at a level as low as $2 \%$ and more than 100 copies $/ 20 \mu \mathrm{l}$ [9]. For such a low proportion, the E-test method missed a resistant strain.

The Seeplex ${ }^{\circledR}$ ClaR-H. pylori ACE detection kit does not allow detection of the A2142C mutation. However, this mutation is less common (usually $<5 \%$ of resistant isolates) $[7,5]$.

As indicated in the Materials and Methods, the Seeplex ${ }^{\circledR}$ ClaR-H. pylori ACE detection kit includes 3 primer pairs with a DPO structure which allows amplification of the $H$. pylori $23 \mathrm{~S}$ rDNA (621 bp amplicon) and detection of the A2142G and A2143G mutations (194 bp and $475 \mathrm{bp}$, respectively). The first primer pair is designed to hybridize regardless of the presence of any mutation inside the PCR fragments. In the case of the A2142G mutation, its specific primer hybridizes and generates a $194 \mathrm{bp}$ PCR product with the reverse H. pylori $23 \mathrm{~S}$ rDNA primer. In the case of the A2143G mutation, its specific primer hybridizes and forms a 475 bp PCR product with the forward H. pylori $23 \mathrm{~S}$ rDNA primer. Therefore, it is not possible to distinguish between 1) gastric biopsies containing a mixture of a wild-type strain and a mutated strain and 2) biopsies containing only a mutated strain (the 621 bp band corresponding to amplification of $H$. pylori $23 \mathrm{~S}$ rDNA is almost always present). It has no practical consequences because the detection of a resistant population is sufficient to exclude macrolides from the eradication therapy to be implemented.

Moreover, for 22 biopsies where A2143G was detected by DPO-PCR, the corresponding $475 \mathrm{bp}$ amplicon was alone in only 4 cases which means that for these corresponding DNAs the reverse $H$. pylori $23 \mathrm{~S}$ rDNA apparently failed to hybridize and to generate the additional $621 \mathrm{bp}$ amplicon. We believe that this is the reason why, in some rare cases, false negatives by DPOPCR can occur (a total of 3 in the present study). The primer pairs which allow the amplification of the H. pylori $23 \mathrm{~S}$ rDNA could be slightly modified to avoid this problem.

\section{Conclusion}

Users should keep in mind that whenever possible H. pylori culture should be performed, and only in cases where standard microbiology fails, the use of molecular methods are really indicated. The rationale behind this is that not only clarithromycin resistance is of interest but also that of other antimicrobials like tetracycline, quinolones, rifamycins and metronidazole. However, the Seeplex ${ }^{\circledR}$ ClaR-H. pylori ACE detection kit is an excellent molecular test to detect $H$. pylori in gastric biopsies and to study its sensitivity to clarithromycin, especially in laboratories without expertise in culturing this bacterium and without a real-time PCR apparatus. At a time when clarithromycin resistance is increasing (prevalence is $>20 \%$ in many countries), clinical laboratories could be enticed by this new PCR format.

\section{Acknowledgements}

The authors thank Leila Labadi and Salha Ben Amor (Université de Bordeaux, Centre National de Référence des Campylobacters et des Hélicobacters, Bordeaux, France) for technical assistance.

\section{Author details}

'Université de Bordeaux, Centre National de Référence des Campylobacters et des Hélicobacters, 146 rue Léo Saignat, 33000 Bordeaux, France. ${ }^{2} \mathrm{CHU}$ de Bordeaux, Hôpital Pellegrin, Laboratoire de Bactériologie, Place Amélie Raba Léon, 33076 Bordeaux cedex, France. ${ }^{3}$ INSERM U853, 33000 Bordeaux, France.

Authors' contributions

$\mathrm{PL}$ and FM analyzed the data and wrote the paper. ES performed the research.

All authors read and approved the final manuscript.

\section{Competing interests}

The authors declare that they have no competing interests.

Received: 26 July 2011 Accepted: 17 October 2011

Published: 17 October 2011

\section{References}

1. Malfertheiner P, Megraud F, O'Morain C, Bazzoli F, El-Omar E, Graham D, Hunt R, Rokkas T, Vakil N, Kuipers EJ: Current concepts in the management of Helicobacter pylori infection: the Maastricht III Consensus Report. Gut 2007, 56(6):772-781.

2. Occhialini A, Urdaci M, Doucet-Populaire F, Bebear CM, Lamouliatte H, Megraud F: Macrolide resistance in Helicobacter pylori: rapid detection of point mutations and assays of macrolide binding to ribosomes. Antimicrob Agents Chemother 1997, 41(12):2724-2728.

3. Menard A, Santos A, Megraud F, Oleastro M: PCR-restriction fragment length polymorphism can also detect point mutation A2142C in the $23 \mathrm{~S}$ rRNA gene, associated with Helicobacter pylori resistance to clarithromycin. Antimicrob Agents Chemother 2002, 46(4):1156-1157. 
4. Agudo S, Alarcon T, Urruzuno P, Martinez MJ, Lopez-Brea M: Detection of Helicobacter pylori and clarithromycin resistance in gastric biopsies of pediatric patients by using a commercially available real-time polymerase chain reaction after Nuclisens semiautomated DNA extraction. Diagn Microbiol Infect Dis 2010, 67(3):213-219.

5. Burucoa C, Garnier M, Silvain C, Fauchere JL: Quadruplex real-time PCR assay using allele-specific scorpion primers for detection of mutations conferring clarithromycin resistance to Helicobacter pylori. J Clin Microbiol 2008, 46(7):2320-2326

6. Cambau E, Allerheiligen V, Coulon C, Corbel C, Lascols C, Deforges L, Soussy CJ, Delchier JC, Megraud F: Evaluation of a new test, genotype HelicoDR, for molecular detection of antibiotic resistance in Helicobacter pylori. J Clin Microbiol 2009, 47(11):3600-3607.

7. Oleastro M, Menard A, Santos A, Lamouliatte H, Monteiro L, Barthelemy P, Megraud F: Real-time PCR assay for rapid and accurate detection of point mutations conferring resistance to clarithromycin in Helicobacter pylori. J Clin Microbiol 2003, 41(1):397-402.

8. Chun JY, Kim KJ, Hwang IT, Kim YJ, Lee DH, Lee IK, Kim JK: Dual priming oligonucleotide system for the multiplex detection of respiratory viruses and SNP genotyping of CYP2C19 gene. Nucleic Acids Res 2007, 35(6):e40

9. Woo HY, Park DI, Park H, Kim MK, Kim DH, Kim IS, Kim YJ: Dual-priming oligonucleotide-based multiplex PCR for the detection of Helicobacter pylori and determination of clarithromycin resistance with gastric biopsy specimens. Helicobacter 2009, 14(1):22-28.

10. Cho AR, Lee MK: [A comparison analysis on the diagnosis of Helicobacter pylori infection and the detection of clarithromycin resistance according to biopsy sites]. Korean J Lab Med 2010, 30(4):381-387.

11. Megraud F: A growing demand for Helicobacter pylori culture in the near future? Ital I Gastroenterol Hepatol 1997, 29(6):574-576.

12. Megraud F, Lehours P: Helicobacter pylori detection and antimicrobial susceptibility testing. Clin Microbiol Rev 2007, 20(2):280-322.

\section{Pre-publication history}

The pre-publication history for this paper can be accessed here: http://www.biomedcentral.com/1471-230X/11/112/prepub

\section{doi:10.1186/1471-230X-11-112}

Cite this article as: Lehours et al:: DPO multiplex PCR as an alternative to culture and susceptibility testing to detect Helicobacter pylori and its resistance to clarithromycin. BMC Gastroenterology 2011 11:112.

\section{Submit your next manuscript to BioMed Central and take full advantage of:}

- Convenient online submission

- Thorough peer review

- No space constraints or color figure charges

- Immediate publication on acceptance

- Inclusion in PubMed, CAS, Scopus and Google Scholar

- Research which is freely available for redistribution

Submit your manuscript at www.biomedcentral.com/submit
Biomed Central 